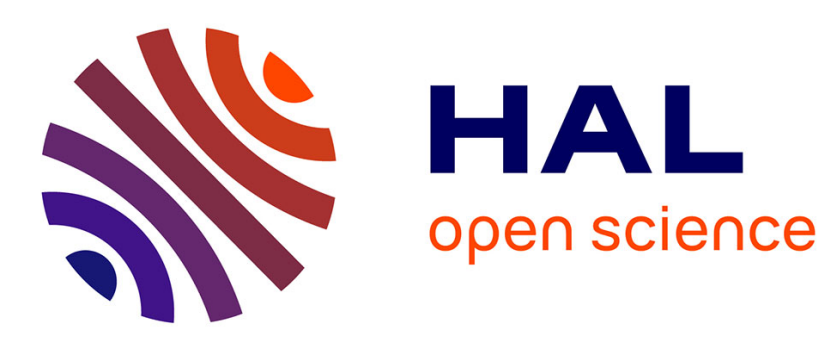

\title{
Logique des homicides dits altruistes. Clinique de l'infanticide
}

\author{
Yohan Trichet, Anne-Sophie Dupont
}

\section{To cite this version:}

Yohan Trichet, Anne-Sophie Dupont. Logique des homicides dits altruistes. Clinique de l'infanticide. Bulletin de psychologie, 2011, 514 (4), pp.347-357. 10.3917/bupsy.514.0347 . hal-01728295

\section{HAL Id: hal-01728295 \\ https://hal.univ-rennes2.fr/hal-01728295}

Submitted on 10 Mar 2018

HAL is a multi-disciplinary open access archive for the deposit and dissemination of scientific research documents, whether they are published or not. The documents may come from teaching and research institutions in France or abroad, or from public or private research centers.
L'archive ouverte pluridisciplinaire $\mathbf{H A L}$, est destinée au dépôt et à la diffusion de documents scientifiques de niveau recherche, publiés ou non, émanant des établissements d'enseignement et de recherche français ou étrangers, des laboratoires publics ou privés. 


\section{LOGIQUE DES HOMICIDES DITS ALTRUISTES. CLINIQUE DE L'INFANTICIDE}

Yohan Trichet, Anne-Sophie Dupont

Groupe d'études de psychologie | « Bulletin de psychologie »

2011/4 Numéro 514 | pages 347 à 357

ISSN 0007-4403

Article disponible en ligne à l'adresse :

https://www.cairn.info/revue-bulletin-de-psychologie-2011-4-page-347.htm

\section{Pour citer cet article :}

Yohan Trichet, Anne-Sophie Dupont « Logique des homicides dits altruistes. Clinique de l'infanticide », Bulletin de psychologie 2011/4 (Numéro 514), p. 347-357. DOI 10.3917/bupsy.514.0347

Distribution électronique Cairn.info pour Groupe d'études de psychologie.

(c) Groupe d'études de psychologie. Tous droits réservés pour tous pays.

La reproduction ou représentation de cet article, notamment par photocopie, n'est autorisée que dans les limites des conditions générales d'utilisation du site ou, le cas échéant, des conditions générales de la licence souscrite par votre établissement. Toute autre reproduction ou représentation, en tout ou partie, sous quelque forme et de quelque manière que ce soit, est interdite sauf accord préalable et écrit de l'éditeur, en dehors des cas prévus par la législation en vigueur en France. Il est précisé que son stockage dans une base de données est également interdit. 


\section{Logique des homicides dits altruistes. Clinique de l'infanticide \\ TRICHET Yohan* DuPONT Anne-Sophie**}

En procédant, en 1821, à l'isolement des suicides homicides dans le cadre de sa réforme nosographique 1, Esquirol rapportait quelques-unes des premières observations d'homicides, dits altruistes de l'histoire de la psychiatrie moderne française. Il conférait un statut psychopathologique à ces passages à l'acte motivés, permettant d'inférer une aliénation mentale chez leurs auteurs. L'influence des vives critiques psychiatrico-judiciaires, adressées à sa doctrine des monomanies, dès les années 1820-1830 et, plus encore, durant la seconde moitié du XIX ${ }^{\mathrm{e}}$ siècle, explique, en partie, l'absence d'étude majeure traitant de ces passages à l'acte durant cette période. Il faudra attendre les premières décennies du $\mathrm{XX}^{\mathrm{e}}$ siècle et l'investigation psychiatrique des pathologies de l'affectivité (Muchielli, 1994 , p. 243) pour que paraissent, en 1913, les études de Charles Vallon et Georges Génil-Perrin sur les homicides par altruisme. Suivront celles de Gaétan Gatian de Clérambault, en 1921, dans lesquelles il formule la thèse d'un trouble fondamental d'anxiété altruiste, à la source de ces homicides. Dans son sillage, son élève Georges Perrussel leur consacra, en 1923, la première thèse de médecine, qui reprenait les notions et les observations du maître. Bien que le discours psychiatrique ait pu, depuis, procéder à quelques apports intéressants, notamment la critique du terme même d'altruisme, il tente d'éclairer les coordonnées de ces passages à l' acte en les chiffrant dans des études épidémiologiques (Biéder, Callens, 2000 ; Chocard, Juan, 2002). Si plusieurs données cliniques concordantes sont repérées par l'ensemble de ces travaux, ils peinent, cependant, à rendre compte de la position subjective de l'auteur de l'infanticide altruiste qui se révèle être, dans la majorité des cas, la mère de l'enfant (Lalou, 1986, p. 194) ${ }^{2}$. C'est précisément cette position subjective, que nous allons tenter de cerner, à partir de l'examen des cas de Claudine et de Mme X. L'hypothèse, développée dans cet article, soutient que ces deux mères ont agi par nécessité psychique, face au réel d'une séparation impossible.

\section{HISTORIQUE}

Si l'on tente de retracer l'histoire des investigations psychiatriques des homicides, dits altruistes, quatre travaux majeurs, dans la description de cette réalité clinique, peuvent être isolés et présentés, selon la perspective chronologique suivante: le suicide homicide d'Esquirol, en 1821, l'homicide par altruisme de Vallon et Génil-Perrin, en 1913 et l'homicide altruiste de Clérambault, en 1921, prolongé par Perrussel en 1923.

\section{Esquirol : le suicide homicide (1821)}

Lors de sa description de la monomanie homicide, dans le cadre de son mémoire consacré au suicide, daté de 1821, Esquirol rapporte quelques observations de «suicide précédé d'homicide » (Esquirol, 1821, p. 276), appelé aussi «suicide homicide » (Esquirol, 1838a, p. 276). D'après lui, «presque tous les suicides homicides sont des lypémaniaques ${ }^{3}$, dominés par une passion portée jusqu'au délire jouissant d'ailleurs de leur raison ;

* Centre hospitalier spécialisé de la Sarthe, laboratoire de psychopathologie et clinique psychanalytique, Université Rennes 2.

** Centre hospitalier spécialisé de la Sarthe, unité de consultations et de soins ambulatoires à la maison d'arrêt Le Mans-Les Croisettes.

Correspondance: Yohan Trichet, Centre hospitalier spécialisé de la Sarthe, 20 avenue du 19 mars 1962, 72703 Allonnes.

$<y$.trichet2@voila.fr>

1. Initiée avec son article «Monomanie» (1819b, p. 114-125).

2. Ce que montrent toutes les études menées sur le sujet. À partir d'une analyse des archives judiciaires, tirées du Compte général de la justice criminelle (1825-1910), Richard Lalou notait qu'au XIX ${ }^{\mathrm{e}}$ siècle, les infanticides, qui faisaient l'objet d'une procédure judiciaire, étaient commis, dans $90 \%$ des cas, par les mères et, à la fin de ce siècle, dans $100 \%$ des cas. Aussi, écrit-il, l'infanticide est « un phénomène féminin ».

3. Sujets présentant une lypémanie. Terme forgé par Esquirol, en 1819, pour désigner «la mélancolie vraie [...] la mélancolie des anciens », c'est-à-dire une monomanie ou «délire partiel sans fièvre, avec crainte et tristesse prolongées » (Esquirol, 1819a, p. 150). 
quelques motifs plus ou moins plausibles à leur sens, les déterminent » (Esquirol, 1838a, p. 276). S'appuyant, à la suite de Pinel, sur la théorie stoïcienne des passions, Esquirol repère deux formes cliniques à ces actes : «Parmi les malheureux qui tuent avant de se suicider, il en est qui obéissent à des passions véhémentes qui les portent promptement à ce double homicide; les autres sont mus par des passions dont les effets sont lents à se produire » (Esquirol, 1838a, p. 280). Au-delà de leur variabilité formelle, Esquirol cerne deux motifs majeurs présidant à leur réalisation : « Il en est qui tuent les personnes qui leur sont les plus chères pour les préserver des peines de la vie, des dangers de la damnation; enfin on en a vu tuer les objets de leur plus vive tendresse, ne voulant pas s'en séparer, croyant être réunis avec eux après la mort » (Esquirol, 1838a, p. 280). Seul le premier motif peut être qualifié d'altruiste, si l'on se rapporte à sa définition. En effet, selon le Dictionnaire historique de la langue française, le terme d'altruisme « désigne la disposition innée de l'être humain à la bienveillance à l'égard des autres membres de sa communauté, et qui coexiste avec l'égoïsme. Sa valeur s'est étendue en morale pour toute conduite et attitude où l'intérêt personnel est subordonné à celui des semblables, sans motivation religieuse $\gg\left(\right.$ Rey, 1992) ${ }^{4}$.

Tuer un être cher, pour le protéger ou pour le rejoindre dans la mort, représente, d'après Esquirol, les deux motifs les plus fréquemment invoqués par les auteurs de ces crimes. Remarquons, également, que la majorité des observations rapportées sont des infanticides commis par les mères et des uxoricides (meurtre de l'épouse par son mari), si bien qu'Esquirol se demande : «Peut-on croire qu'une pareille violation des premières lois de la nature, que tant d'exaltation de l'imagination, que tant d'égarement de la sensibilité, puisse se concilier avec la plénitude de la santé, avec l'intégrité de la raison? Ne faut-il pas, au contraire, être arrivé au dernier degré du délire pour se déterminer à tuer une femme que l'on chérit, des enfants qu'on adore ? [...] et cependant plusieurs faits prouvent que ces malheureux, hors de cet acte, avant et après son accomplissement, sont calmes et raisonnables. Ce calme, cette raison ne s'observent-ils pas chez ces maniaques qui, pour le plus léger motif, pour la contrariété la plus inoffensive, vont se livrer aux actes de fureur la plus aveugle ? (Esquirol, 1838a, p. 280). Au nom d'un état normal précédant le crime, Esquirol opère un rapprochement entre les suicides homicides et la manie sans délire décrite dès 1798 par Pinel (Trichet, Lévy, 2008), de sorte

4. Le terme d'altruisme a vraisemblablement été créé par Auguste Comte vers 1830. Il est attesté depuis 1852. qu'il les appréhende comme une espèce de monomanie homicide. Si la dynamique et l'émergence de l'acte l'incitent à ce rapprochement, il ne s'en tient pas là. Le cadre nosographique des suicides homicides est large, car Esquirol y inclut certains homicides passionnels, suivis de suicide, du type délire de jalousie, et les suicides réciproques. Or, la majeure partie des cas qu'il présente se rapporte à la lypémanie. Ainsi, Esquirol inaugure, probablement, le rapprochement, toujours d'actualité, entre homicide altruiste et lypémanie, notre moderne mélancolie. Selon lui, Platner et Michel Ethmuller avaient, déjà, «rapporté quelques faits de monomanie homicide qu'ils nomment mélancolie homicide ; c'est, disent-ils, un trouble de l'esprit sans égarement de la raison» (Esquirol, 1838b, p. 342).

En outre, Esquirol s'intéressant au vécu subjectif des auteurs de ces crimes, souligne que, majoritairement, «ils commettent l'homicide avec calme, tranquillité, au moins en apparence : après l'avoir consommé, ils ne sont point émus ni inquiets; ils sont plus calmes après l'avoir commis qu'auparavant, quelques fois ils paraissent contents. Plusieurs vont faire la déclaration de leur crime à la police, aux tribunaux, ou en parlent à ceux qu'ils rencontrent : loin de se dérober aux recherches, ils attendent qu'on les arrête, ils demandent à subir la peine capitale. Le suicide, précédé d'homicide, est ordinairement aigu ; cependant quelque fois il est chronique, et offre tous les caractères d'un acte réfléchi et volontaire » (Esquirol, 1838a, p. 276). Esquirol avait déjà repéré, dans ces lignes, certains traits caractéristiques de la clinique de l'homicide, dit altruiste, que l'on retrouve dans le cas de Pierre Rivière ${ }^{5}$ (Foucault, 1973) ou dans celui, récent, d'Andréa Yates (Salecl, 2003).

\section{Vallon et Génil-Perrin : l'homicide par altruisme (1913)}

Lors de son étude des actes d'altruisme morbide, défini comme une «pathologie de l'émotion tendre » ${ }^{6}$ (Ribot cité par Génil-Perrin, 1910, p. 233), en 1910, Génil-Perrin décrit un altruisme criminel, qui fera l'objet, deux ans plus tard, d'un examen détaillé. Réalisé avec son confrère de Sainte-Anne, Charles Vallon, cet examen montre que l'altruisme criminel met en évidence la présence de sentiments altruistes chez les auteurs de certains attentats contre la société, la propriété et les personnes. Bien que repérés de longue date,

5. Esquirol fut consulté, avec d'autres sommités médicales de l'époque, fondatrices, en 1829, des Annales d'hygiène publique et de médecine légale, afin d'attester de l'aliénation mentale de Pierre Rivière et de le soustraire, ainsi, à la peine de mort.

6. Génil-Perrin préfère le syntagme de «déviation de l'émotion tendre » (1910, p. 234). 
soulignent Génil-Perrin et Vallon, ces faits cliniques n'avaient donné lieu à aucun travail d'ensemble et, encore moins, rapportés à un «déterminisme psychopathique » (Vallon, GénilPerrin, 1913a, p. 83).

C'est dans le cadre des attentats contre les personnes, que Génil-Perrin et Vallon en viennent à étudier les actes de violence et d'homicides par altruisme. Procédant à leur classification, ils les répartissent en trois catégories, à savoir, l'altruisme des criminels, l'homicide par altruisme, commis dans l'intérêt d'un tiers et, enfin, l'homicide par altruisme, commis dans l'intérêt supposé de la victime. Indiquons les termes de la première catégorie: à l'opposé du caractère monstrueux et égoïste de leurs actes, certains criminels font preuve, dans leur existence, de sentiments ou d'émotions altruistes. Les auteurs n'insistent guère sur la seconde occurrence, les homicides par altruisme, commis dans l'intérêt d'un tiers, car ils leur paraissent "généralement moins complexes et moins intéressants que les autres au point de vue psychiatrique » (Vallon, Génil-Perrin, 1913a, p. 95). De sorte qu'ils vont particulièrement analyser la troisième catégorie, l'homicide par altruisme, commis dans l'intérêt supposé de la victime, dont ils recensent six modalités : les meurtres altruistes des mélancoliques, des persécutés, des fanatiques, les cas d'interprétation douteuse, la question de l'euthanasie et l'automutilation indirecte.

$\mathrm{Au}$ terme de leur étude, Génil-Perrin et Vallon en arrivent à la conclusion que les passages à l'acte examinés "présentent tous ce caractère commun que, dans la genèse de chacun d'eux, l'idée altruiste et l'émotion tendre ont joué un rôle tantôt prépondérant, tantôt plus effacé, mais toujours indéniable » (Vallon, Génil-Perrin, 1913b, p. 184). Aussi, estiment-ils que le déterminisme psychologique ne porte pas sur la nature de l'émotion, mais sur son intensité ou, plus précisément, sur la défaillance d'une «synthèse mentale » (Vallon, GénilPerrin, 1913b, p. 187) censée, lorsqu'elle est affermie, brider les émotions. Rapportant cette défaillance à la théorie de la dégénérescence, encore dominante à l'époque, Génil-Perrin et Vallon considèrent que les auteurs de crimes altruistes appartiennent au groupe des dégénérés et, spécialement, à la classe des émotifs. Bien différente sera notre perspective, centrée sur une clinique du sujet.

\section{Clérambault : l'homicide altruiste (1921)}

Un siècle après l'article d'Esquirol et sa description du suicide homicide, Clérambault rapporte, dans son article éponyme, consacré à l'homicide altruiste, chez les mélancoliques [1921], deux observations de tentative ou menace d'infanticide altruiste, suivi de suicide. Il s'agit de deux mères. A. Angelina-Marie a tenté de tuer sa fille unique et, ensuite, de se défenestrer et $\mathrm{P}$. Marie-Josèphe a proféré des menaces de suicide collectif. Tentant de cerner les déterminants subjectifs de tels passages à l'acte ou de leurs projets, l'auteur postule, chez le sujet, l'existence d'une anxiété altruiste, dont le paradigme est l'anxiété maternelle. À partir de nombreuses observations, notamment les deux rapportées dans son article, Clérambault dégage deux lois principales, auxquelles une troisième peut être adjointe.

Il formule, ainsi, la première loi de l'anxiété altruiste: «Dès qu'un délirant souffre d'Anxiété Altruiste, on est en droit d'appréhender de sa part l'Homicide altruiste. C'est une loi déjà démontrée que la peur de la mort engendre l'impulsion suicide ; thanatophobie et suicide sont pratiquement une équation » (Clérambault, 1921, p. 674). Autre formulation de cette première loi : «Le sujet qui craint un danger pour lui-même doit le craindre également pour ses proches » (Clérambault, 1921, p. 677).

La seconde loi porte sur la logique du passage à l'acte : «Quiconque est anxieux pour autrui peut tuer, et logiquement doit tuer» (Clérambault, 1921, p. 675). Ou encore il décrète «que de l'Anxiété Altruiste sortent logiquement l'idée de l'Homicide Altruiste et l'impulsion à l'accomplir » (Clérambault, 1921, p. 675). Le passage à l'acte est tellement logique pour Clérambault qu'il affirme que «ne pas interner un malade souffrant d'anxiété altruiste, ne pas le séparer de ses proches qu'il aime le plus, serait une faute lourde. Le danger de l'anxiété altruiste pour celui qui en est l'objet est proportionnel à l'affection dont il procède, et cela encore en vertu d'une logique normale » (Clérambault, 1921, p. 675). Il insiste sur ce point: «La logique normale à elle seule porte l'anxieux altruiste au meurtre altruiste » (Clérambault, 1921, p. 676). Se trouve, ainsi, signifié que l'acte n'est pas engendré par une logique morbide, déterminée par des illogismes apparents, mais par une anxiété altruiste, dont la structure s'apparente à une certitude délirante. Située en amont de l'acte, elle correspond au postulat altruiste de l'homicide. Elle apparaît comme une holophrase, définie par Lacan comme «l'absence d'intervalle entre S1 et S2 » (Lacan, 1964, p. 215), corrélative d'une prise en masse signifiante du réel et, de fait, non dialectisable.

Enfin, une troisième loi peut être repérée sous la plume de Clérambault : "Le délirant tend d'autant plus à tuer ses proches qu'il craint d'en être séparé » (Clérambault, 1921, p. 677).

\section{Perrussel : l'homicide altruiste des mélanco- liques et des persécutés (1923)}

La thèse de George Perrussel sur les homicides altruistes ne possède guère d'originalité qui lui soit 
propre, sinon sa primauté historique. Elle est, en effet, la thèse princeps sur le sujet. Inutile de dire que cette thèse doit beaucoup au travail de Clérambault, non seulement par son titre et ses observations (une vingtaine provenant du maître, comme l'indique Perrussel, 1923), mais, aussi, par sa perspective psychopathologique. Son auteur, qui fut interne des Asiles de la Seine et de l'Infirmerie spéciale, dirigée par Clérambault, y développe les thèses du maître.

Son principal intérêt réside dans les observations cliniques qu'elle contient. Jamais ouvrage n'en avait rapportées autant. Ce sont, essentiellement, des cas d'infanticides altruistes, proportion qui semble représentative de la clinique à en croire l'auteur. D'un autre côté, il note que, dans onze cas sur cinquante, le sujet tue « les êtres aimés [qui] ne peuvent pas se passer de lui » (Perrussel, 1923, p. 57). Dans la réalité des faits, il s'agit, le plus souvent, d'une mère, qui recourt à ce type d'acte, devant son impossibilité à se séparer de ses enfants. Une telle constatation avait déjà été faite par Esquirol et Clérambault. Compte tenu de son importance dans la logique de l'infanticide altruiste, elle mérite d'être examinée.

\section{CLINIQUE DE L'HOMICIDE ALTRUISTE : DEUX CAS D'INFANTICIDES}

$\mathrm{Si}$, dans la littérature spécialisée, tous les meurtres d'enfants, commis par la mère, étaient traditionnellement regroupés sous le même terme d'infanticide, on peut, désormais, remarquer une nette tendance à les séparer en deux catégories. Il est fort probable que les travaux de Phillip. J Resnick aient participé, de manière décisive, à l'établissement de cette bipartition en néonaticide et filicide (Resnick, 1969). Cet auteur propose d'appeler néonaticides les infanticides d'enfants de moins de vingt-quatre heures. D'autres réservent le terme d'infanticide à ces actes qui seraient, très fréquemment, le fait de jeunes femmes en situation de précarité. Elles ne tenteraient, ensuite, que très rarement, de se suicider (Resnick, 2009). D'un autre côté, Resnick désigne par filicides les infanticides d'enfants de plus de vingt-quatre heures. À sa suite, certains distinguent même les filicides précoces, lorsque l'enfant a moins d'un an, des filicides tardifs, au-delà d'un an (Dayan, Andro, Dugnat, 1999, p. 386-387). Ces actes, que d'autres appellent libéricides, seraient, tendanciellement, perpétués par des femmes plus âgées, ayant déjà élevé au moins un enfant (Resnick, 1969) et présentant un état dépressif majeur (Dayan et coll., 1999, p. 386 ; Ravit, Roman, 2009). Si les premiers seraient, très nettement, commis pour des motifs de grossesse non désirée, $83 \%$, selon une étude impliquant 38 cas d'infanticides, essentiellement issus du milieu psychiatrique (Resnick, 1969), les seconds seraient, souvent, motivés par des sentiments altruistes, $56 \%$ des cas selon la même étude. Cette donnée clinique, concordante avec les premières descriptions d'Esquirol, également réalisées en milieu psychiatrique, doit être relativisée, selon des études plus récentes, faites à partir d'échantillons de la population générale. Dans celles-ci, le mobile de la maltraitance a pris, alors, une importance majeure (Resnick, 2009). Néanmoins, les infanticides n'en constituent pas moins une horreur et une énigme pour l'opinion publique et la raison commune. La présence régulière, dans les faits divers, d'infanticides, comme l'affaire Courjault (2006) en témoigne.

Dans son effort de rendre intelligible ces passages à l'acte, c'est-à-dire dans sa tentative d'en circonscrire le réel et les enjeux subjectifs, le clinicien s'intéresse aux ressorts psychiques de ces mères, qui sacrifient leur progéniture pour leur bien ou celui d'autrui. Quels liens unissent la mère à son enfant? Et au père de son enfant ? Car, comme le soulignent J.-L. Viaux et S. Combaluzier, «l'analyse de dynamique entre les deux parents de l'enfant victime est indispensable à la compréhension de ce qui se joue dans l'infanticide » (Viaux, Combaluzier, 2010, p. 14).

Si une logique préside à cette réalité clinique, comme l'affirme Clérambault, il semble qu'elle doit être corrélée à la position subjective de la mère meurtrière. Nous tenterons de le montrer, grâce à l'étude de deux cas cliniques. Dans le premier, il s'agit d'une tentative d'infanticide, au bénéfice du père des enfants et, dans le second, d'un infanticide, au bénéfice de l'enfant, victime du délire de sa mère.

\section{Le cas de Claudine}

Nous allons essayer, maintenant, de cerner, chez Claudine, les conditions d'émergence et la logique du postulat d'altruisme, qui fut à la source de sa tentative d'infanticide et de suicide.

Depuis la mort de sa mère, en 1987, Claudine est hospitalisée régulièrement pour état mélancolique, avec prégnance d'idées suicidaires. En 1991, au cours d'une phase de stabilisation, comme elle émet le souhait de rentrer au domicile familial, son mari demande le divorce. Elle est, depuis lors, hospitalisée à temps complet. Son dossier médical mentionne de nombreux symptômes, dont une autodépréciation majeure et un profond sentiment d'inutilité. Lorsque j'intègre le service, en 2002, elle souhaite me rencontrer, car, dit-elle, «ça fait du bien de parler». Elle ne participe à aucune activité. Elle reste allongée sur son lit toute la journée ou bien regarde la télévision. À chaque fois qu'un projet lui est proposé, Claudine se mutile les bras. 
Elle parle toujours d'un ton lent et monocorde et se présente sous le signifiant « dépression ». Aussi, au regard de la clinique, aucune perspective de sortie ne peut être sérieusement envisagée.

\section{La mort aux rats}

Lors du troisième entretien, Claudine évoque la rencontre avec son ex-mari, la naissance de leurs enfants et sa souffrance de ne plus les voir. Elle justifie la décision de sa famille d'avoir rompu tout contact avec elle en alléguant sa maladie, son passage à l'acte et les motifs de celui-ci : «C'est normal si mes enfants ne veulent plus me voir, ils n'ont pas eu une enfance facile, avec ma maladie, ils ont dû faire le travail à la maison pour aider leur père [...]. C'est normal si mon mari m'en veut parce qu'un jour, j'ai voulu empoisonner mes enfants. Un soir, peu de temps après la mort de ma mère, je voulais en finir. J'ai préparé la soupe pour tous mes enfants. Mon mari ne rentrait qu'à vingt-trois heures, donc lorsqu'il reviendrait de son travail, on serait tous morts. Je ne voulais pas laisser mes enfants avec leur père ça lui aurait fait trop de soucis. J'étais dans la cuisine et Michaël [son fils aîné] est arrivé, il a vu la mort aux rats, il m'a demandé ce que je voulais faire. Michaël m'a dit que c'était une mort lente et douloureuse alors que je pensais que c'était instantané [...]. Je regrette, j'aurais dû le faire pour moi toute seule. »

Elle dit avoir tout prémédité. Il ne s'agissait, donc, nullement, d'un acte impulsif, mais d'un acte idéalisé, sous-tendu par l'idée du bien pour son mari : « ça lui aurait fait trop de soucis ». La réalisation d'un tel projet aurait constitué un infanticide altruiste, suivi de suicide, ce qu'Esquirol désignait en 1821 du nom de suicide homicide, comme nous l'avons vu.

Afin de mieux saisir ce qui opère, pour Claudine, dans cette tentative de passage à l'acte, il nous faut prendre en compte les circonstances du déclenchement de sa psychose. Cliniquement, elle décompense en suite du décès de sa mère. Le rapprochement entre ces deux événements, décès de sa mère et décompensation mélancolique, nous amène à penser qu'elle prend la place de sa mère comme malade. Cette «maladie» va se traduire par une forte inhibition. Figée dans un profond état d'abattement, elle reste au lit toute la journée. En outre, elle refuse de s'alimenter et ressent une très grande culpabilité. Puis, le décès de son père, un an plus tard, renforcera sa symptomatologie mélancolique.

\section{La terreur du père}

$\mathrm{Au}$ cours des séances, Claudine décrit un père qu'elle a toujours craint, le qualifiant même de «dangereux et pervers». Elle livre deux scènes marquantes de son enfance : "Une fois mon père a étalé tous les couteaux de cuisine sur la table.
J'étais terrifiée, je ne savais pas ce qu'il allait pouvoir faire, il pouvait peut-être nous tuer. » Puis, ajoute-t-elle, « lorsque j'avais neuf ans, mon père est rentré, il était énervé, il a pris sa carabine, s'est assis sur le lit, a tenu le fusil à côté du berceau. J'étais dans le lit avec ma sœur, je tremblais de peur. Je n'ai pas dormi de la nuit, j' avais peur qu'il s'en serve ». Elle relate, également, la fréquente violence du père à l'encontre de la mère, qui se manifestait, essentiellement, par des agressions verbales (cris, insultes) et physiques (objets renversés, cassés), sans, pour autant, qu'il ne portât de coups ni sur elle ni sur sa famille. Dès lors, elle s'est toujours donné pour mission, de protéger sa mère, ainsi que ses frères et sœurs, de son père. Ce rôle protecteur l'a soutenue dans l'existence. Or, après le décès de son père, le couple parental se retrouve dans le même caveau. La séparation de ses parents, tant désirée par Claudine, mais toujours refusée par sa mère, n'est plus possible. Enterrés dans le même caveau, leur union est désormais éternelle. Le père est représenté comme le père jouisseur, que rien ni personne ne peut arrêter, pas même la mort.

La vision du caveau, prévu pour ses deux parents, dès l'enterrement de sa mère, lui était déjà apparue insupportable. Nous entrevoyons, dans l'effet de cette découverte, jusqu'où se déploie, pour elle, la toute-puissance du père : «Au cimetière, ils avaient creusé pour deux, mais je ne voulais pas enterrer ma mère avec mon père : il lui avait fait trop de mal. Une fois morte, ma mère avait le droit au repos [...], mais même mort, il pourrait peut-être continuer à lui faire du mal [...]. Je me suis sentie impuissante pendant toute cette période où ma mère était malade. Je me suis sentie responsable de sa mort».

\section{La mère : l'impossible séparation}

La réunion de ses parents dans le même caveau lui est insupportable pour une autre raison: son père vient rompre, dans le réel, la relation fusionnelle qu'elle entretenait avec sa mère. En effet, en tant qu'aînée de la fratrie, Claudine a toujours secondé et même suppléé sa mère. Une fois partie de la maison, elle a continué à l'aider et à la soutenir dans l'éducation de ses frères et sœurs. Lorsque l'état de santé de sa mère, atteinte d'un cancer, s'est dégradé, Claudine l'a hébergée pour s'en occuper.

Ainsi, Claudine et sa mère ont fait couple et, d'une certaine manière, ce couple fonctionne encore après la mort, puisque sa mère, dont la perte n'a pas été symbolisée, fait retour dans le réel par le biais d'hallucinations visuelles : « Le soir je vois ma mère morte, je la vois à côté de moi, comme si c'était la réalité. Elle m'accompagne toujours ». Ainsi, la mort n'est pas synonyme de séparation 
pour Claudine : elle et sa mère ne font qu'Un. Ces éléments cliniques nous amènent à penser que sa mère constituait un autre elle-même. Avec le décès de celle-ci, Claudine perd l'appui imaginaire sur lequel elle se réglait dans l'existence. Ce laisser tomber maternel la confronte à un impossible à supporter, auquel elle répond par une tentative de suicide infanticide.

En miroir à son vécu subjectif, elle a la certitude que, comme son père, son mari ne peut pas s'occuper de ses enfants, et que, par conséquent, ces derniers ne peuvent pas vivre sans leur mère. Il est vrai qu'au décès de sa mère, son père ne s'est pas occupé de son dernier fils. C'est Claudine qui l'a élevé quelque temps. De même, elle est persuadée que son mari ne peut pas s'occuper de leurs enfants, formulation imaginaire, puisque, depuis son absence du foyer conjugal, c'est son mari qui élève seul leurs quatre enfants. Pour protéger le mari, elle n'a, donc, d'autre solution, tel est son postulat délirant, que de les tuer. Il y a donc une disjonction entre la place occupée par son mari auprès de leurs enfants et sa certitude délirante. Son postulat altruiste émerge du défaut de nouage entre les instances de la paternité, comme en réponse à la question du Père qui se pose à elle, avec la perte de sa mère. La logique de ce postulat implique la carence du père symbolique et non celle du père réel.

En tentant de se tuer et de tuer ses enfants, elle répond à un impossible de la séparation entre elle, sa mère et ses enfants, qui, du fait de ce défaut de séparation, sont en position d'objets.

\section{La faute du sujet}

Si nous poursuivons la perspective développée jusqu'ici, nous pouvons considérer les automutilations de Claudine comme une tentative d'inscrire, sur son corps, une séparation d'avec la mère. Cette séparation n'étant pas symbolisée, cette tentative se réitère, c'est-à-dire ne cesse pas de ne pas s'écrire. Faisons l'hypothèse qu'il s'agit là, pour Claudine, du point de réel de sa position mélancolique. En effet, à propos de ces coupures, elle nous dit «c'est comme une pulsion». Elle ne les interprète pas, et n'en dit pas grand-chose, ce qui suggère qu'elles ne constituent pas un appel à l'Autre. Les coupures font l'impasse sur la symbolisation. Elle en éprouve une jouissance impossible à contrôler : " Je ne peux pas m'en empêcher ». Dans un sens, ses automutilations présentifient le châtiment qu'elle attend pour ne pas avoir protégé sa mère, ne pas l'avoir séparée de son mari dans la vie comme dans la mort et pour avoir tenté d'empoisonner ses enfants ; à leur propos, elle a pu dire : «tant pis pour moi, bien fait pour moi». Claudine a la certitude délirante d'avoir commis une faute qui ne renvoie nullement au sentiment de culpabilité du névrosé. Elle doit en payer le prix sous la forme de coupures et d'auto-accusations : «Je me sens coupable, je n'aurais pas dû laisser ma mère dans le même caveau que mon père, et j'aurais dû faire quelque chose pour m'interposer entre eux. Je me sens coupable de la maladie de ma mère $[. .$.$] coupable de ne pas avoir assez protégé$ ma mère. »

\section{La mort du sujet}

À deux reprises Claudine a été confrontée au réel. Le décès de sa mère a constitué un laisser tomber, entraînant sa propre chute. Par sa radicalité, cette dernière dévoile l'identification de Claudine à l'objet déchet. Puis, au cimetière, le trou, creusé pour ses parents, a présentifié, pour elle, la réunion de ses parents pour l'éternité, soit une occurrence du rapport sexuel, la jouissance devenant alors possible et illimitée. Pour le sujet névrosé, les rapports entre les sexes sont réglés par l'asymétrie structurale entre les positions féminines et masculines, de sorte qu'il y a, toujours, ratage de l'objet, ce que Lacan formule ainsi : «il n'y a pas de rapport sexuel » (Lacan, 1975, p. 17). Or, pour le sujet psychotique, la jouissance de l'objet est possible, ainsi que nous venons de l'indiquer pour Claudine. L'une des conséquences cliniques du rapport sexuel est un retour de la jouissance, aux effets mortifères évidents, assimilables à une mort du sujet. Celle-ci correspond à «l'abolition des effets de signification promus par la métaphore paternelle »(Fridman, Millas, 1997, p. 92). Du vide de la signification abolie, a émergé, chez Claudine, une signification altruiste, qui l'a conduite à sa tentative de suicide infanticide. On peut même considérer que ses multiples hospitalisations, ses coupures et sa clinophilie manifestent une forme de disparition du sujet. Elle incarne, alors, l'objet déchet, ravalée, ainsi, par une jouissance que rien ne semble pouvoir border.

\section{Mme X}

$\mathrm{Si}$, dans de nombreux cas de néonaticides, les mères ne pouvaient vivre avec leur nouveau-né, l'acte se révélant être ainsi une solution à "l'impossible face-à-face » (Viaux, Combaluzier, 2010, p. 11), il n'est pas rare que les infanticides altruistes soient commis par des mères, au nom d'une impossibilité, pour leurs enfants, de vivre sans elles. La tentative de suicide altruiste de Claudine découle de cette certitude. On la discerne, également, dans l'observation de Mme X, rapportée par Patricia Johansson-Rosen. Sa patiente lui affirma, dans l'après-coup de son acte - elle a tué sa fille aînée par pendaison, puis a essayé, à l'aide de médicaments, de se tuer avec sa deuxième fille - : «Des enfants ne peuvent pas vivre sans leur mère » (Johansson-Rosen, 1987, p. 35). Lors 
de son incarcération, Mme $\mathrm{X}$ tente à plusieurs reprises de se suicider. Elle est, alors, hospitalisée dans un service de psychiatrie. Le lendemain, apparaît un état mélancolique faisant craindre un nouveau passage à l'acte suicidaire. Avec raison, l'auteur classe ce passage à l'acte parmi les suicides altruistes. En reprenant les moments-clés de l'existence de Mme X, nous allons essayer de mettre en perspective son passage à l'acte avec son fonctionnement psychique.

\section{Le premier épisode à seize ans : la mort de sa} mère

Mme X est née au Vietnam. Quatrième d'une fratrie de sept enfants, elle est l'aînée de deux filles. Peu avant de mourir, sa mère avait obtenu de son mari sa stérilisation, de sorte qu'ils ne puissent plus avoir d'enfants. Par ce sacrifice, annihilant toute paternité future, elle pensait avoir l'assurance qu'il assume la charge de leurs enfants. Son père demande, alors, à Mme X, de quitter l'école, afin de remplacer sa mère au foyer. Il se montre violent avec elle, il l'accuse de le voler. En suite du décès de sa mère, elle est profondément abattue et perd le goût de vivre. Ainsi que le souligne JohanssonRosen, «la perte de sa mère est incontestablement le drame de la vie de cette patiente » (JohanssonRosen, 1987, p. 36).

\section{La rencontre du mari et son lien à ses enfants}

À vingt-et-un ans, elle rencontre un officier français : «C'est le coup de foudre», dit-elle (Johansson-Rosen, 1987, p. 36). Il vit en France et elle au Vietnam. Aussi entretiennent-ils une abondante correspondance. Une grève du courrier postal interrompant leur lien épistolaire, Mme X sombre, à nouveau, dans un état dépressif sévère, qui s'estompe très rapidement avec la reprise du courrier. Son profond abattement laisse supposer que le lien imaginaire est prévalent pour elle : il constitue un rempart précaire à l'effondrement subjectif. Deux ans plus tard, elle quitte sa famille pour l'officier rejoindre et l'épouser. Le couple a deux enfants en peu de temps. Pendant ses grossesses, elle «n'est plus seule au monde» (Johansson-Rosen, 1987, p. 36). Les accouchements ont été vécus sans difficulté, d'autant, confie-t-elle, qu'elle ne s'est « jamais totalement séparée de ses filles » (Johansson-Rosen, 1987 , p. 36). Elle se décrit comme une mère très anxieuse pour ses enfants, point qui concorde avec la seconde loi formulée par Clérambault. Il fait, en effet, de l'anxiété maternelle, le paradigme de l'anxiété altruiste, dans laquelle s'enracine l'homicide altruiste. En outre, il convient de souligner que Mme X était considérée par ses proches, nous dit l'auteur, comme une mère incomparable. Il s'agit-là d'une donnée relativement fréquente dans la clinique des infanticides. Bien souvent, ces mères sont présentées, par leur entourage, comme des mères modèles, de sorte que la découverte ou la révélation de leurs crimes ou tentatives de crimes possèdent un caractère irréel et énigmatique.

Qu'elles soient classiquement présentées, par leur entourage, comme des mères modèles ou bien qu'elles se présentent, elles-mêmes, avec cette « sorte de faux moi », pouvant servir, à l'occasion, de béquille imaginaire compensatrice, comme a pu le remarquer A. Henry dans sa pratique pénitentiaire (Henry, 2009, p. 89).

\section{Le déclenchement de cet épisode mélancolique}

L'état dépressif de cette femme s'est déclenché cinq mois avant son passage à l'acte meurtrier, en suite du départ de sa voisine, une amie proche, partie vivre avec son compagnon. En proie à des insomnies, elle reproche à celle-ci de l'avoir quittée. Surgissent des idées de mort, consécutives au laisser en plan de cette amie, qui lui servait de béquille imaginaire. Quelques temps après, son mari, malade et déprimé consécutivement à une promotion professionnelle, lui annonce qu'elle ne doit plus compter sur lui. Elle se retrouve seule, « lâchée par tous », souligne l'auteur (JohanssonRosen, 1987, p. 35). Elle subit un double laisser tomber de l'autre, évocateur du « laissé en plan » de Schreber (Schreber, 1903, p. 61). Émergent des idées hypochondriaques relatives à son mari et ses filles. Un délire se forme : elle est persuadée que les absents sont morts. À cette époque, débute une troisième grossesse, que le couple souhaite interrompre. Une fausse couche mettra fin à cette grossesse non désirée. Peu après, Mme $\mathrm{X}$ ouvre, au domicile familial, le robinet de gaz, destinant, ainsi, sa famille à disparaître. La tentative échoue : une fenêtre était restée ouverte.

Un soir, elle déclare à son mari, qui s'apprêtait à effectuer un déplacement : «Un homme comme toi, on ne peut rien lui faire, tout le monde doit l'aimer. » Au moment où elle prononce ces paroles, elle avait déjà décidé de tuer leurs deux filles. La phrase énoncée prend une signification particulière lorsqu'elle avoue : "J'ai voulu tuer mes enfants pour amener mon mari à se tuer ». Son passage à l'acte interviendra peu après.

\section{Son hospitalisation}

Du fait de ses passages à l'acte infanticides et suicidaires, Mme X est hospitalisée. Elle présente un état d'aboulie, auquel succède un bref épisode hallucinatoire visuel et verbal. Elle voit les corps de ses deux filles allongés côte à côte sur un lit. Elle est également confuse, elle pense être chez elle en train de faire à manger dans sa cuisine. Peu de temps après, l'annonce d'un non-lieu psychiatrique, clôturant la procédure pénale dont elle faisait l'objet, Mme X écrit une lettre, où elle 
s'accuse d'avoir joué la folle et d'avoir trompé le personnel soignant et les experts. Son état psychique s'améliore, elle exprime de nouveaux projets, souhaite reprendre la vie conjugale et s'occuper de sa fille.

\section{Le déclenchement du passage à l'acte : logique de l'acte}

Reprenons les moments décisifs dans son existence, dans la perspective du déclenchement de sa psychose. Mme X décompense un épisode mélancolique, au moment où sa voisine, considérée comme un «substitut maternel », déménage pour rejoindre son amant (Johansson-Rosen, 1987, p. 37). On peut se demander, avec l'auteur, s'il ne fait pas irruption, comme Un-père, dans ce couple imaginaire, que la patiente formait avec sa voisine. On en déduit que cet épisode mélancolique résulterait d'une conjonction de facteurs déclenchants : perte de la béquille imaginaire et irruption d'Un-père. Quelques temps après, le mari s'effondre et se met hors-circuit. Mme X se trouve, à nouveau, confrontée à la même situation que celle vécue au moment du décès de sa mère, lorsqu'elle avait seize ans. La disparition de celle-ci avait déclenché son premier épisode mélancolique. Le départ de la voisine renvoie au décès de sa mère et le laisser tomber de son mari à celui de son père. Par sa requête de stérilisation, adressée à son mari, la mère de Mme X avait, ainsi, implicitement énoncé qu'on ne peut compter sur le père que lorsqu'il est castré. Sa demande, sinon sa commande, signifie que, pour elle, la fonction de père n'est pas compatible avec l'exercice de la paternité dans la réalité. Pour que son mari puisse exercer sa paternité, c'est-à-dire, comme un père réel, s'occuper de leurs enfants, il doit accepter de ne plus pouvoir être père.

Le départ de sa voisine, puis la mise hors-circuit de son mari, constituent des liegen lassen (Johansson-Rosen, 1987, p. 37), soit des laisser tomber de l'autre, le partenaire imaginaire. Cette situation déclenchante favorise le dévoilement de sa position subjective, d'identification à l'objet $a$. Les coordonnées du déclenchement de la psychose de Mme X sont homologues, dans sa configuration structurale, à son premier épisode dépressif, vécu au moment du décès de sa mère. Elle rencontre un réel, dont elle ne peut se protéger qu'au prix de la vie de sa fille aînée.

\section{Qui et que visait-elle? À la fois son mari et elle-même}

\section{- Elle visait son mari}

Incontestablement, cette femme visait son mari : «J'ai voulu tuer mes enfants pour amener mon mari à se tuer ». Ainsi se dévoile la haine qu'elle éprouvait pour son mari. On peut faire un parallèle entre le sacrifice demandé par la mère au père, sacrifice d'une livre de chair ou objet $a$ visant la procréation des enfants à venir, sacrifice d'être père et le sacrifice de la préférée de son mari. C'est le père des enfants qui est visé, à travers la mise à mort de la fille aînée. La mère de Mme $\mathrm{X}$ visait son mari, lorsqu'elle lui a demandé de sacrifier ses organes reproducteurs (vasectomie) et, donc, sa fonction de père et d'éventuels enfants. Elle lui impose de faire le sacrifice d'une paternité à venir, c'est-à-dire de sacrifier son désir de paternité dans le réel: à l'époque, son père accepte, puis s'effondre (Johansson-Rosen, 1987, p. 42).

Lors de son infanticide, Mme $\mathrm{X}$ vise son mari en tuant sa préférée et souhaite, ainsi, l'amener à se suicider. De manière plus radicale que sa mère, elle souhaite par son acte faire disparaître son mari, le père de ses enfants : elle vise le sacrifice du père réel. Car enfin, ne l'a-t-il pas abandonnée en se mettant hors-circuit? Ne s'est-il pas séparé d'elle, la laissant alors en plan? Nous pourrions alors évoquer la troisième loi formulée par Clérambault selon laquelle le sujet tue l'être cher devant la crainte d'en être séparé. Plus précisément, dans le cas de Mme X, le passage à l'acte répond à la séparation.

En outre, le postulat ou la signification de son délire - «Un enfant ne peut pas vivre sans sa mère » (Johansson-Rosen, 1987, p. 37) - renvoie à l'absence du Père symbolique dans sa fonction séparatrice, au trou qu'il occupe dans la chaîne signifiante et à une signification de jouissance de l'Autre maternel. Pour la mère de Mme X, le père n'est bon qu'à être castré dans le réel et pour Mme X qu'à se suicider. Pour cette dernière, le Père n'était pas déjà mort. D'où ce stratagème sacrificiel.

\section{- Elle se visait comme objet $a$}

Si une telle logique régissant son acte repose sur sa propre identification imaginaire à sa mère, il semble que ces infanticides procèdent, également, d'une autre visée. En parlant de son acte comme d'un suicide, Mme X indique une autre logique à son acte : en tuant et en voulant tuer ses enfants, c'est elle-même qu'elle vise. Griesinger avait déjà repéré la portée suicidaire de tels actes. Dans nombre de cas, affirme-t-il, « l'acte commis a réellement le caractère d'une mutilation, en quelque sorte d'un suicide que ces individus font contre eux-mêmes en en tuant un autre, puisque ce malheureux père qui aimait tendrement son enfant veut, en le frappant, se frapper lui-même » (Griesinger, 1865, p. 316-317).

Pour Mme X, ses enfants sont des doubles d'ellemême : elle confie ne s'être «jamais totalement séparée de ses filles ». C'est ici que l'on trouve 
implicitement la dimension altruiste. En effet, s'il n'y a pas explicitement une connotation altruiste de son acte, on la déduit, cependant, de la logique de celui-ci et de ses dires : ses propres filles lui paraissent à son image, c'est-à-dire, effondrées. Aussi, elle tue ses doubles pour les délivrer de son effondrement, c'est-à-dire de sa propre mort subjective, qu'elle projette sur elles. Elle fusionne avec ses filles en incarnant le Un de la jouissance. Elle court-circuite l'Autre du signifiant. On peut même avancer que cette fusion imaginaire est subordonnée à la fusion signifiante de l'holophrase, propre à caractériser la structure psychotique.

Les rapports du sujet à ses semblables se trouvent, dès lors, soumis au tranchant mortel du transitivisme imaginaire, où l'autre et lui ne font qu'un. Ainsi que le soulignait J.-C. Maleval, les cadavres ont une fonction de miroir du sujet, «ce que les "suicides altruistes" de mélancoliques viennent confirmer : ils tuent parfois leurs proches avant de se supprimer eux-mêmes, parce qu'ils les perçoivent à leur image dans un état d'extrême déchéance » (Maleval, 1989, p. 129). À cet égard, le cas rapporté par Charles Vallon et Georges Génil-Perrin est exemplaire. «Un monsieur, souffrant d'idées hypocondriaques très accusées, rencontre dans une rue à Stockholm, un enfant qui lui ressemblait. De cette ressemblance physique, le monsieur conclut à la possibilité d'une ressemblance morale et s'imagine que l'enfant est appelé à souffrir un jour les mêmes tourments que luimême. Pour le mettre à l'abri de ce triste destin, il sort un revolver de sa poche et le tue. » (Vallon, Génil-Perrin, 1913a, p. 103). Les explications du meurtrier, auxquelles les deux auteurs font allusivement référence, illustrent le lien imaginaire unissant le sujet à l'objet sacrifié. En se reconnaissant dans cet enfant croisé dans la rue, cet homme se trouve en proie au surgissement du double, témoignant de son aliénation imaginaire ou engluement au miroir: me tuer en tuant l'autre qui me ressemble apparaît comme la solution subjective. C'est sur son double, que s'exerce la haine du sujet, haine ressortissant de sa propre identification à l'objet déchet. Contrairement au paranoïaque, lui aussi englué dans l'imaginaire, mais localisant la jouissance dans l'Autre, le sujet mélancolique tend, lui et ses doubles, à incarner l'objet $a$.

\section{De l'impossible séparation}

Pour cette femme, il est patent que l'imaginaire possède une fonction compensatrice. En effet, elle s'appuie sur l'imaginaire, c'est-à-dire sur un lien à un semblable, pour parer à la carence de la fonction paternelle. Mais son enfermement dans l'imaginaire est subordonné à son identification à l'objet déchet, non chu de l'image spéculaire. Abandonnée par sa voisine, comme elle l'a été par sa mère, ses propres filles, dont on peut supposer qu'elle ne s'est jamais séparée, lui apparaissent effondrées comme elle-même.

Les coordonnées des passages à l'acte ou tentatives de passages à l'acte sacrificiels ne s'éclairent qu'à les rapporter au cadre familial dans le lien du sujet à ses parents, à son mari et à ses enfants. Autrement dit, ces actes dépendent de son rapport à l'Autre. À la mort de sa mère, Mme $\mathrm{X}$ répond par un effondrement mélancolique, d'où émerge, vraisemblablement, comme l'indique Alain Vaissermann, le «germe» d'une «impossible séparation » (Vaissermann, 1987, p. 40), qui fera retour dans le réel, tel un phénomène élémentaire, au moment du lâchage par sa voisine, puis par son mari. Surgit, alors, la certitude que ses enfants ne pourront vivre au-delà de sa mort, tout comme elle a dû se sentir mourir au moment du décès de sa propre mère. Pour elle, la séparation est impossible, elle ne renvoie à aucune perte fondatrice, structuralement opérée par l'aliénation du sujet au langage et, ce faisant, elle constitue un point de réel indépassable.

\section{POUR CONCLURE}

Le syntagme d'homicide altruiste comporte un paradoxe et, même, un non-sens, car il suppose l'existence de l'Autre, soit une instance symbolique, dont l'incomplétude, corrélée avec celle du sujet (\$) fonde l'altérité. Or, les sujets, qui commettent ces crimes, ne sont pas séparés de l'Autre. Aussi peut-on les qualifier de sujets non divisés. L'examen des deux cas cliniques présentés dans le cadre de cet article suggère que ces actes interviennent comme une réponse du sujet confronté au réel d'une séparation impossible, c'est-à-dire comme une solution soumise à une logique singulière. En effet, l'homicide dit altruiste se révèle déterminé par un postulat altruiste dont les formes cliniques diffèrent selon que le supposé bénéficiaire soit la victime ou bien un tiers. Cette logique donne une assise nosographique à ces actes, de sorte que la référence historique à la mélancolie, depuis la lypémanie d'Esquirol se trouve ici fondée ${ }^{7}$. Et si plusieurs études s'accordent à montrer l'existence d'un lien prévalent entre crimes dits altruistes et dépression (Bénézech, 1991), il semble, cliniquement, nécessaire de discerner plus finement, derrière la catégorie mésusée de dépression, une mélancolie psychotique. À cet égard, J.-L Viaux et S. Combaluzier invoquent «une sorte de mélancolie meurtrière» dans nombre de cas de

7. Exceptés les cas où la signification altruiste d'un homicide est produite par le sujet au cours du procès délirant comme ce fut le cas pour Louis (Trichet, Marion, à paraître). 
néonaticides (Viaux, Comabaluzier, 2010, p. 11). De manière plus cruciale, cette logique permet de cerner la position subjective de leur auteur. Elle éclaire ce à quoi il vise.

Dans le crime paranoïaque, le sujet dépose dans l'Autre «le kakon de son propre être ${ }^{8}$ (Lacan, 1946, p. 175). Il peut l'atteindre et le tuer, en frappant une figure de l'Autre jouisseur. L'acte opère, alors, comme une castration dans le réel, dont les effets libératoires sont, parfois, indéniables: le sujet peut en renaître différent ${ }^{9}$. En effet, l'acte est susceptible d'introduire une discontinuité dans l'existence du sujet et, ce faisant, de modifier son rapport à la jouissance. Dans l'homicide, dit altruiste, le sujet identifie dans un autre, non plus symbolique, mais imaginaire, souvent un enfant, sa propre déchéance, son être de déchet, qui l'écrase. Il fait, alors, Un avec son objet, avec son double.

8. Cette notion de kakon a été introduite par C. von Monakow dans les années 1910. Dans son ouvrage, co-écrit avec R. Mourgue, elle désigne aussi bien «le malheur, le danger» (Monakow von, Mourgue, 1928, p. 287) que «sous sa forme primitive, une angoisse actuelle» (p. 291). Elle fut introduite, dans le champ psychiatrique français, en 1931, par Paul Guiraud.

9. Monakow et Mourgue avaient déjà signalé, en 1928, la portée libératrice de ce qu'ils désignèrent comme « crise du kakon» (Monakow, Mourgue, 1928, p. 271).
Par son suicide précédé d'homicide, le sujet « accomplit son destin de kakon », note J.-A Miller (Miller, 1993, p. 9). Si le suicide (ou sa tentative) est fréquent dans les crimes altruistes, il succède très rarement aux crimes paranoïaques. Leurs logiques respectives divergent notamment car il y a de l'Autre dans la paranoïa: un Autre réel «gourmand de l'objet $a »$ (Miller, 1991, p. 11), certes, mais qui n'est pas sans ancrage symbolique. Lacan n'affirmait-il pas, à la fin des années 1970, que « dans la paranoïa, le signifiant représente un sujet pour un autre signifiant» (Lacan, 1977, p. 12). Cependant, le signifiant n'est pas le meurtre de la Chose, d'où la propension du sujet paranoïaque à frapper «la Chose, le kakon, en l'Autre » (Miller, 1991, p. 9). En revanche, dans la mélancolie, où sont traditionnellement classés les crimes altruistes, « la désaliénation radicale de la chaîne signifiante, le rejet de l'inconscient, laisse le sujet en prise directe avec l'objet réel » (Deffieux, 2004, p. 54). Les deux cas, que nous avons examinés, montrent que les victimes ou personnes visées sont, en réalité, des doubles du sujet : l'imaginaire touchant au réel d'une impossible séparation d'avec l'objet de jouissance. Dans sa déchéance, corrélative de son identification à l'objet $a$, le sujet se frappe à travers ses objets, puis se suicide, lorsque aucune autre solution ne s'offre à lui.

\section{RÉFÉRENCES}

BÉNÉZECH (Michel).- Dépression et crime. Revue de la littérature et observations originales, Annales médicopsychologiques, 149, 2, 1991, p. 150-165.

BIÉDER (Joseph), CALlENS (H.).- L'homicide altruiste chez les mélancoliques, Annales médico-psychologiques, 158, 9, 2000, p. 703-708.

Clérambault (Gaëtan Gatien de).- L'homicide altruiste chez le mélancolique [1921], Euvres psychiatriques, Paris, Frénésie éditions, 1998, p. 668-678.

Chocard (Anne-Sophie), JuAN (F.).- Les meurtressuicides. Revue de littérature, Forensic, 12, 2002, p. 47-52.

DAYAN (Jacques), ANDRo (Gwenaëlle), Dugnat (Michel).- Psychopathologie de la périnatalité, Paris, Masson, 1999.

DEFFIEUX (Jean-Pierre).- Le risque suicidaire, La Cause freudienne, 58, 2004, p. 49-55.

Esquirol (Jean-Étienne-Dominique).- Mélancolie, Dictionnaires des sciences médicales, XXXII, Paris, Panckoucke, 1819a, p. 147-183.
ESQUIROL (Jean-Étienne-Dominique).- Monomanie, Dictionnaires des sciences médicales, XXXIV, Paris, Panckoucke, 1819b, p. 114-125.

EsQuirol (Jean-Étienne-Dominique).- Suicide, Dictionnaire des sciences médicales, LIII, Paris, Panckoucke, 1821, p. 213-283.

Esquirol (Jean-Étienne-Dominique).- Suicide, Des maladies mentales considérées sous les rapports médical, hygiénique et médico-légal [1838a], I, Paris, Frénésie, 1989, p. 259-331.

EsQUIROL (Jean-Étienne-Dominique).- Mémoire sur la monomanie homicide, Des maladies mentales considérées sous les rapports médical, hygiénique et médicolégal [1838b], II, Paris, Frénésie, 1989, p. 335-360, p. 342.

Foucault (Michel).- Présentation. Moi, Pierre Rivière, ayant égorgé ma mère, ma sœur et mon frère, Paris, Gallimard, 1973.

Fridman (Pablo), Millas (Daniel).- L'exaltation maniaque, dans Collectif, Le conciliabule d'Angers, Paris, Agalma, 1997, p. 89-97. 
GÉNIL-PERRIN (Georges).- L'altruisme morbide, L'année psychologique, XVII, 1910, p. 223-250.

GRIESINGER (Wilhelm).- Traité des maladies mentales. Pathologie et thérapeutique, Paris, A. Delahaye, 1865.

Guiraud (Paul).- Les meurtres immotivés, L'évolution psychiatrique, 4, 2, 1931, p. 25-34.

HenRy (Anne).- Suivis de femmes infanticides à la prison de Rennes, Enfances \& psy, 44, 2009, p. 84-91.

Johansson-Rosen (Patricia).- Une mère mélancolique, meurtrière de son enfant, dans Actes de la cause freudienne, XIII, 1987, p. 35-37.

LACAN (Jacques).- Propos sur la causalité psychique [1946], Écrits, Paris, Le Seuil, 1966, p. 151-193.

LACAN (Jacques).- Le séminaire, livre XI. Les quatre concepts fondamentaux de la psychanalyse, 1964, Paris, Le seuil, 1973.

LACAN (Jacques).- Le séminaire, livre XX, Encore, 1972-1973, Paris, Le seuil, 1975

LACAN (Jacques).- Ouverture de la Section clinique, Ornicar? Revue du champ freudien, 9, 1977, p. 7-14.

LALOU (Richard).- L'infanticide devant les tribunaux français (1825-1910), Communications, 44, 1986, p. 175-200.

Maleval (Jean-Claude).- Le champ passionnel de la psychose, L'évolution psychiatrique, 54, 1, 1989, p. 115-135.

MiLler (Jacques-Alain).- Clinique ironique, Revue de la cause freudienne, 23, 1993, p. 7-13.

Monakow (Constantin von), Mourgue (Raoul).Introduction biologique à l'étude de la neurologie et de la psychopathologie, Paris, Félix Alcan, 1928.

Muchielli (Laurent).- Renouvellements et déplacements dans le champ criminologique au $\mathrm{XX}^{\mathrm{e}}$ siècle, dans Muchielli (L.), Histoire de la criminologie française, Paris, L'harmattan, 1994, p. 240-247.
PERRUSSEL (Georges).- L'homicide altruiste des mélancoliques et des persécutés, Thèse, Paris, Jouve et $\mathrm{C}^{\mathrm{ie}}, 1923 . \mathrm{i}$

RAVIT (Magali), Roman (Pascal).- Clinique de l'infanticide. Un corps à corps mortifère, Psychologie clinique et projective, $X V, 2009$, p. 145-178.

RESNICK (Philip J.).- Meurtre de nouveau-né : une synthèse psychiatrique sur le néonaticide [1969], Enfance \& psy, 44, 3, 2009, p. 42-54.

RESNICK (Philip J.).- Retour sur les néonaticides : perspectives actuelles, Enfance \& psy, 44, 3, 2009, p. 55-59.

REY (Alain).- Dictionnaire historique de la langue française, Paris, dictionnaires Le Robert, 1992.

SCHREBER (Daniel-Paul).- Mémoires d'un névropathe [1903], Paris, Le seuil, 1975.

SALECL (Renata).- Le réel du crime : une mère infanticide, Savoirs et cliniques, 2, 2003, p. 41-51.

TRICHET (Yohan), LÉvy (Alexandre).- Scarification et (auto-)mutilation dans la psychose, L'information psychiatrique, 84, 5, 2008, p. 395-401.

TRICHET (Yohan), MARION (Élisabeth).- De la monstruosité du matricide à l'élaboration d'un altruisme délirant, L'évolution psychiatrique (à paraître).

VAISSERMANN (Alain).- Discussion, dans Actes de la cause freudienne, XIII, 1987, p. 40.

VALlon (Charles), GÉNIL-PERRIN (Georges).- Crime et altruisme, Archives d'anthropologie criminelle de médecine légale et de psychologie normale et pathologique, 230, 1913a, p. 81-110.

VALlon (Charles), GÉNIL-PERrin (Georges).- Crime et altruisme (suite et fin), Archives d'anthropologie criminelle de médecine légale et de psychologie normale et pathologique, 230, 1913b, p. 161-187.

Viaux (Jean-Luc), Combaluzier (Serge).- Néonaticide, un non-désir mélancolique : étude clinique de 12 cas, L'évolution psychiatrique, 75, 1, 2010, p. 3-17. 